\title{
Falhas em transformadores de potência: Estudo de caso do Sutf6-03 na Uhe Samuel
}

\author{
Power transformer failures: Sutf6-03 Case study at Uhe Samuel \\ Fallos del transformador de potencia: Caso práctico sutf6-03 en Uhe Samuel
}

\author{
Helano Oliveira Góes \\ ORCID: https://orcid.org/0000-0003-0438-1181 \\ Universidades Federal de Rondônia, Brasil \\ E-mail: helanogoes@gmail.com \\ Fábio Herrera Fernandes \\ ORCID: https://orcid.org/0000-0003-3242-8303 \\ Centro Universitário São Lucas, Brasil \\ E-mail: fabio26012002@gmail.com \\ Rafael Luis da Silva \\ ORCID: https://orcid.org/0000-0002-9485-479X \\ Centro Universitário São Lucas, Brasil \\ E-mail: rafael.luis@saolucas.edu.br \\ Alex Gomes Pereira \\ ORCID: https://orcid.org/0000-0003-3563-4161 \\ Centro Universitário São Lucas, Brasil \\ E-mail: alexgp885@gmail.com \\ Marcus Vinicius Oliveira Braga \\ ORCID: https://orcid.org/0000-0001-9965-8125 \\ Centro Universitário São Lucas, Brasil \\ E-mail: marcus.braga@saolucas.edu \\ Renato Lima dos Santos \\ ORCID: https://orcid.org/0000-0001-8306-3821 \\ Centro Universitário Aparício Carvalho FIMCA, Brasil \\ E-mail: renato.fbt@gmail.com
}

\begin{abstract}
Resumo
Os transformadores de potência estão entre os equipamentos de maior importância no sistema elétrico e são fundamentais para transmissão e distribuição de energia elétrica, para tanto há a necessidade de ser monitorados e analisados rigorosamente. Considerando a importância deste equipamento, este estudo buscou analisar a ocorrência no transformador elevador SUTF6-03 da Usina Hidrelétrica Samuel, o qual foi retirado de operação devido a atuação das proteções diferenciais. A partir dos dados gerados devidos à falha no transformador, foi realizado o estudo de caso, para coleta e análise de dados dos relatórios enviados pelo Laboratório Central da Eletronorte, das amostras de óleo do transformador, onde esses dados foram compilados em forma de gráficos. Fez-se uma pesquisa sobre a teoria de transformadores e os métodos de diagnóstico de faltas por meio da análise de óleo. Este trabalho mostra que é possível por meio do Método de Rogers diagnosticar o motivo da falta ocorrida no transformador.
\end{abstract}

Palavras-chave: Análise de óleo; Método de Rogers; Transformadores de potência; Usina Hidrelétrica Samuel; Central Hidroeléctrica Samuel.

\begin{abstract}
Power transformers are among the most important pieces of equipment in the electrical system and are fundamental for the transmission and distribution of electrical energy, for which there is a need to be monitored and analyzed rigorously. Considering the importance of this equipment, this study sought to analyze the occurrence in the elevator transformer SUTF6-03 of the Samuel Hydroelectric Power Plant, which was removed from operation due to the performance of the differential protections. Based on the data generated due to the failure of the transformer, a case study was carried out to collect and analyze data from the reports sent by Eletronorte's Central Laboratory, of the transformer oil samples, where these data were compiled in the form of graphs. Research was done on the theory of transformers and fault diagnosis methods through oil analysis. This work shows that it is possible through the Rogers Method to diagnose the reason for the fault that occurred in the transformer.
\end{abstract}

Keywords: Oil analysis; Rogers method; Power transformers; Samuel Hydroelectric Plant; Samuel Hydroelectric Power Station.

\section{Resumen}

Los transformadores de potencia se encuentran entre los equipos más importantes del sistema eléctrico y son fundamentales para la transmisión y distribución de energía eléctrica, por lo que es necesario monitorearlos y analizarlos rigurosamente. Considerando la importancia de este equipo, este estudio buscó analizar la ocurrencia en el 
transformador elevador SUTF6-03 de la Central Hidroeléctrica Samuel, el cual fue retirado de operación debido al desempeño de las protecciones diferenciales. A partir de los datos generados por falla del transformador, se realizó un estudio de caso para recolectar y analizar los datos de los informes enviados por el Laboratorio Central de Eletronorte, de las muestras de aceite del transformador, donde estos datos fueron recopilados en forma de gráficos. Se investigó la teoría de los transformadores y los métodos de diagnóstico de fallas a través del análisis de aceite. Este trabajo muestra que es posible a través del Método Rogers diagnosticar el motivo de la falla que ocurrió en el transformador.

Palabras clave: Análisis de aceite; Método de Rogers; Transformadores de poder; Central Hidroeléctrica Samuel; Central Hidroeléctrica Samuel.

\section{Introdução}

O transformador é um dispositivo que através da ação de um campo magnético converte a energia elétrica de uma dada frequência e nível de tensão, em energia elétrica de mesma frequência, mas em outro nível de tensão, sendo esse maior ou menor, dependendo da sua aplicação (Chapman, 2013). O transformador de potência deve aliar no seu processo de construção as seguintes características: boa regulação de tensão; alto rendimento; baixas correntes; baixas perdas (Jordão, 2002).

Outrossim, os transformadores de potência são equipamentos de alto custo para usinas de energia elétrica, circunspecto pela elevação da tensão do gerador, propiciando a transmissão de energia do mesmo para linhas de transmissão e subestações, onde a eletricidade é distribuída e entregue aos centros consumidores. Nessa perspectiva, o monitoramento dos transformadores de potência é imprescindível para o excelente funcionamento da usina.

O transformador é um equipamento que opera sob condições adversas tais como: campos elétricos elevados, temperatura de trabalho elevada, exposição direta ao ambiente, entre outras. Além disso, algumas situações eventuais solicitam grandemente a resistência do maquinário como por exemplo: sobretensões de manobra, descargas atmosféricas, correntes de curto-circuito (Aguiar, 2007).

Para Becharag (2010), anomalias no fornecimento de energia elétrica implicam em graves aspectos, de forma, que há requisitos específicos para qualidade de energia, continuidade de fornecimento e disponibilidade de equipamentos para atendimento às necessidades do sistema elétrico. Tais aspectos, associados a demandas crescentes, limitação de espaço, economia de materiais, classes de tensão mais elevadas, influenciam de forma decisiva no estudo de metodologias que permitam a obtenção de informações precisas sobre tipos de falhas em equipamentos, suas causas, seus desdobramentos, surgimento de falhas e medidas preventivas que possam ser tomadas para minimizar ocorrências futuras de natureza similar.

De acordo com Barbosa (2013), a escolha adequada da técnica empregada para avaliar os dados monitorados, pode resultar na redução da quantidade de sensores, influenciando diretamente no investimento do sistema. Normas técnicas são geralmente utilizadas na avaliação e na coleta de dados, com finalidade de prover eficientemente uma caracterização do ambiente interno do equipamento. Além disso, no sistema elétrico de potência, existem vários métodos usados no acompanhamento do funcionamento do transformador. Os métodos através da análise de óleo do transformador são usados por grandes empresas já consolidadas no mercado.

Aguiar (2007) diz que, um dos méritos das técnicas de monitoramento e diagnóstico é justamente estimar o estado físico do equipamento através de medidas indiretas. Por causa da importância e do seu custo, os transformadores sãos os principais alvos dessas técnicas que visam identificar todos os possíveis defeitos que possam retirar o transformador de operação, sem que o mesmo seja aberto. Com base nisto, fez-se um estudo de caso baseado no sinistro ocorrido no transformador elevador SUTF603 da Usina Hidrelétrica Samuel.

Através da técnica de análise de gás dissolvido (DGA, do inglês Dissolved Gas Analysis), utilizou-se o Método de Rogers para encontrar a causa da falta do transformador. Este estudo tem como objetivo descrever o sinistro ocorrido no transformador SUTF6-03 da Usina Hidrelétrica Samuel, bem como as suas causas. Os objetivos específicos são: Descrever a 
teoria de Transformadores de Potência, apontar os métodos de análise de óleo mais utilizados para diagnóstico e monitoramento de transformadores, comprovar a eficácia do Método de Rogers para diagnóstico de faltas de transformadores.

\section{Metodologia}

Existe uma grande variedade de métodos de diagnósticos de transformadores, bem como, uma grande quantidade de mecanismos para essa finalidade. Equipamentos que vão desde as mais simples funções, como as de medição de temperatura de óleo isolante, até as mais complexas como o monitoramento dos níveis de descargas parciais com o transformador em funcionamento (Bechara, 2010). Em vista disso, o óleo no transformador também é usado com eficiência no seu resfriamento. Para (Almeida, 2020) essa eficiência se baseia no fato de que o líquido, em contato com a parte ativa do transformador, aquece, tornando-se menos denso que o líquido menos aquecido. O óleo menos denso é deslocado para cima.

Esse deslocamento força as camadas superiores a se deslocaram para baixo. Enquanto o transformador está em operação, o óleo mineral e outros materiais dielétricos presentes, ficam submetidos às ações de temperatura e tensões elétricas, passando assim, por processos de decomposição química que resultam na formação de gases (Benedet, 2007).

Além disso, para (Lupi Filho, 2012), a análise de desempenho do transformador por meio do exame das condições do óleo isolante, é uma ferramenta importante para a manutenção preditiva do equipamento. Em decorrência da constante alteração das propriedades do óleo mineral isolante, a vida útil dos transformadores consiste basicamente na proteção do papel isolante do ataque da água e dos compostos ácidos.

Ainda por cima (Lima, 2008), frisa que é necessário o entendimento dos fenômenos físico químicos para um diagnóstico de possíveis efeitos que levam a falhas de operação do transformador. Grande parte dos transformadores tem como base no seu sistema de isolação o óleo. A degradação dos materiais de isolação pode encurtar o tempo de vida útil do transformador.

Sendo o transformador um equipamento de alto custo e de vital importância para o sistema elétrico, faz-se necessário o uso de métodos para diagnósticos de falhas de operação no transformador. Os métodos usados para análise de óleo e os seus resultados seguem padrões ou normas específicas. Dentre as mais utilizadas estão, IEEE - Institute of Electrical and Electronics Engineers, IEC - International Electrotechnical Commission, NBR - Norma Brasileira.

DGA (Dissolved Gas Analysis) ou análise de gases dissolvidos é a principal técnica usada no monitoramento de falhas em equipamentos isolados a óleo. Ela é feita por meio da cromatografia gasosa. A amostra de óleo é coletada e levada para o laboratório, onde é feita a cromatografia gasosa e posteriormente é feita a análise de acordo com os resultados. Para (Silva, 2016), pode-se resumir esse processo de análise em quatro etapas: $1^{\circ}$ Extração do óleo, $2^{\circ}$ Extração dos gases presentes no óleo, $3^{\circ}$ Análise da mistura dos gases extraídos, $4^{\circ}$ Interpretação dos dados.

Em conformidade com (Ikeshoji, 2020), a metodologia usada na interpretação da análise de gases dissolvidos no óleo mineral se baseia na natureza e na quantidade relativa de cada gás. Os métodos mais utilizados para o DGA são: método do gáschave (recomendado pela IEEE C57.104), método Clássico de Rogers, método sugerido pela NBR 72724 e IEC 599, método de Dörnenburg, triângulo de Durval, método da soma dos gases dissolvidos.

De acordo com o (IEEE, 2009), os procedimentos operacionais que devem ser usados na detecção de faltas de transformadores são: Detectar a geração de gases que ultrapassem as quantidades normais de TCG (total de gases combustíveis) e seguir as normas apropriadas para que a possível anormalidade possa ser reconhecida o mais cedo possível, visando de minimizar danos ou evitar uma falha; avaliar o impacto de uma falta no transformador, seguindo as diretrizes ou recomendações das normas reguladoras, e executar a ação recomendada, reduzindo a carga no transformador ou realmente removendo a unidade de serviço.

O óleo do transformador se decompõe por meio da energia que é liberada pela falha. A forma como essa energia é liberada gera uma certa quantidade de gás, e essa quantidade de gás é característica de tipos de falhas específicas (Costa, 2018). 
A interpretação deste método se baseia no fato de que, quando ocorre uma falha no transformador, a concentração de gases associados a cada falha, superam os valores de degradação da isolação do equipamento, valores esses que são estabelecidos de acordo com a norma seguida (Enriquez, 2020). Gases chaves são gases encontrados no óleo dos transformadores e que são usados para determinação de tipos de falhas (IEEE, 2009). Esses gases se dividem em três grupos: hidrocarbonetos e hidrogênio, óxidos de carbono, e gases não oriundos de faltas (Ikeshoji , 2020).

As características físico-químicas são acompanhadas por meio de ensaios periódicos com a função de monitorar a degeneração do óleo, identificando o grau de influência de contaminantes (ácidos, partículas sólidas em suspensão, água e borra) em suas propriedades dielétricas. A principal função da análise físico-química é avaliar quantitativa e qualitativamente os produtos oriundos de degradação presente no óleo. Esse método também avalia processos que aceleram o envelhecimento do equipamento, tais como agentes de oxidação, umidade, entre outros. O processo de coleta deve ser realizado cuidadosamente, evitando a contaminação do recipiente de amostra, o que alteraria o resultado da análise e conduziria à conclusão incorreta sobre a qualidade do óleo e a confiabilidade do equipamento (Senna, 2010).

De acordo com (Mayor, 2020), uma das formas confiáveis de diagnóstico do transformador se dá por meio do controle de concentração dos gases furanos $\mathrm{C}_{4} \mathrm{H}_{4} \mathrm{O}$. Outrossim, a cromatografia gasosa é um método que se baseia na análise físicoquímica por separação de compostos químicos. A separação acontece quando é injetado na coluna um volume conhecido de analito gasoso ou líquido. Um gás denominado gás carregador movimenta as moléculas do analito por meio da coluna. Essa movimentação é inibida pela adesão das moléculas do analito nas paredes da coluna. A progressão das moléculas na coluna depende da força de adesão, que depende do tipo de molécula e do material na fase estacionária (Leopoldino, 2011).

Além disso, as substâncias são extraídas da coluna separadamente, se dissolvem no gás de arraste passando por um dispositivo detector que gera um pulso elétrico proporcional à quantidade de material fluido. $\mathrm{O}$ registro desse pulso em função do tempo é o cromatograma, onde as substâncias apresentam picos com área proporcional à sua massa, possibilitando assim a análise quantitativa (Caldeira, 2019).

Como também, a técnica que utiliza câmeras especiais que fazem a medição da energia infravermelha irradiada pelos objetos sem que seja necessário o contato físico. Permite o monitoramento do comportamento térmico pela determinação e quantificação de calor dos equipamentos (Senna, 2010). O monitoramento termográfico (Figura 1) permite antecipadamente a detecção de faltas, dando mais disponibilidade nos sistemas industriais e nos equipamentos. Nos sistemas elétricos a termografia possui uma relevância maior, pois a temperatura é um dos parâmetros constantemente monitorados, pois a elevação da temperatura é um fator causador de falhas (Santana, 2018).

Os transformadores possuem termômetros analógicos ou digitais para monitoramento da temperatura do óleo e dos enrolamentos. Para o acompanhamento do óleo, o equipamento possui sensores imersos no líquido isolante. No caso dos enrolamentos, essa medição é feita por meio do TC (transformador de corrente) de imagem térmica, que estima a temperatura das bobinas. Atualmente, existem sistemas com maior tecnologia que usam fibras ópticas, com a vantagem de que elas apresentam dados reais da temperatura do enrolamento (Bechara, 2010). 
Figura 1. Imagem de um transformador pelas lentes de uma câmera termográfica.

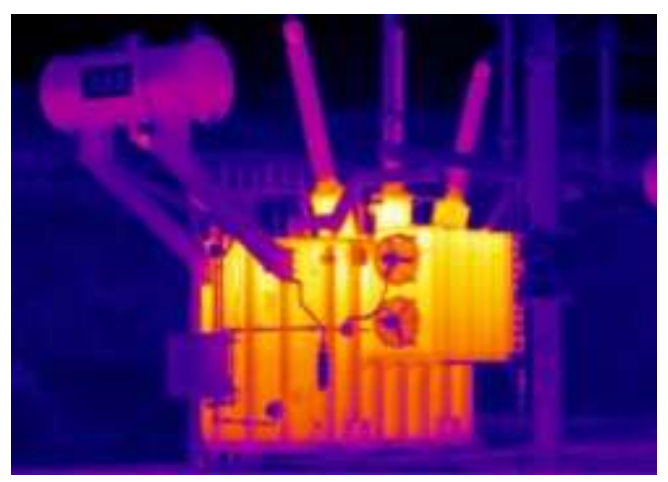

Fonte: Pinterest (2020).

\section{Resultados e Discussão}

A Figura 2 mostra as concentrações de $\mathrm{H}_{2}, \mathrm{CH}_{4}, \mathrm{C}_{2} \mathrm{H}_{2}, \mathrm{C}_{2} \mathrm{H}_{4}, \mathrm{C}_{2} \mathrm{H}_{6}$ e $\mathrm{CO}$, respectivamente. Analisando o gráfico da Figura 2 (a), é possível verificar que ao longo das amostras, os valores de hidrogênio aumentam consideravelmente entre 01/11/2017 e 17/04/2018. Na Figura 2 (b) observa-se que os valores de metano estavam bem abaixo dos valores de referência, tendo seu aumento entre 01/11/2017 e 17/04/2018. Na Figura 2 (c) nota-se uma elevação do valor da concentração do acetileno se o valor da concentração do acetileno se dá entre 01/11/2017 e 17/04/2018. Dando continuidade na análise dos resultados, na Figura 2d observa-se um processo do crescimento da concentração de etileno se inicia entre 01/11/2017 e 17/04/2018. Enquanto que na Figura 2 (e) os valores de etano sempre se mantem bem abaixo dos valores de referência. E por último, a Figura 2 (f) verifica-se que entre 30/07/2012 e 01/08/2013 o iniciou-se uma alta nos valores de monóxido de carbono ficando acima dos valores de referência.

Figura 2. Amostras coletadas das concentrações do transformador SUTF6-03: a) Hidrogênio; b) Metano; c) Acetileno; d) Etileno; e) Etano; f) Etano; e, g) Carbono.

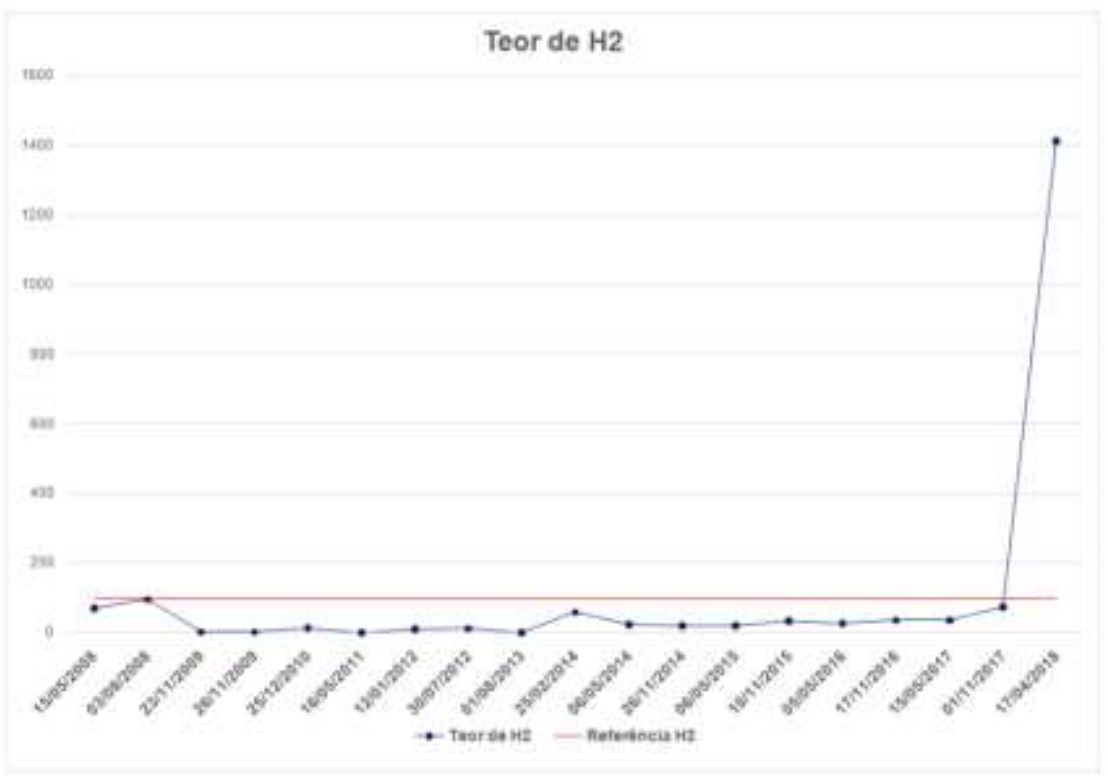

a) 
Research, Society and Development, v. 10, n. 6, e18510615460, 2021 (CC BY 4.0) | ISSN 2525-3409 | DOI: http://dx.doi.org/10.33448/rsd-v10i6.15460

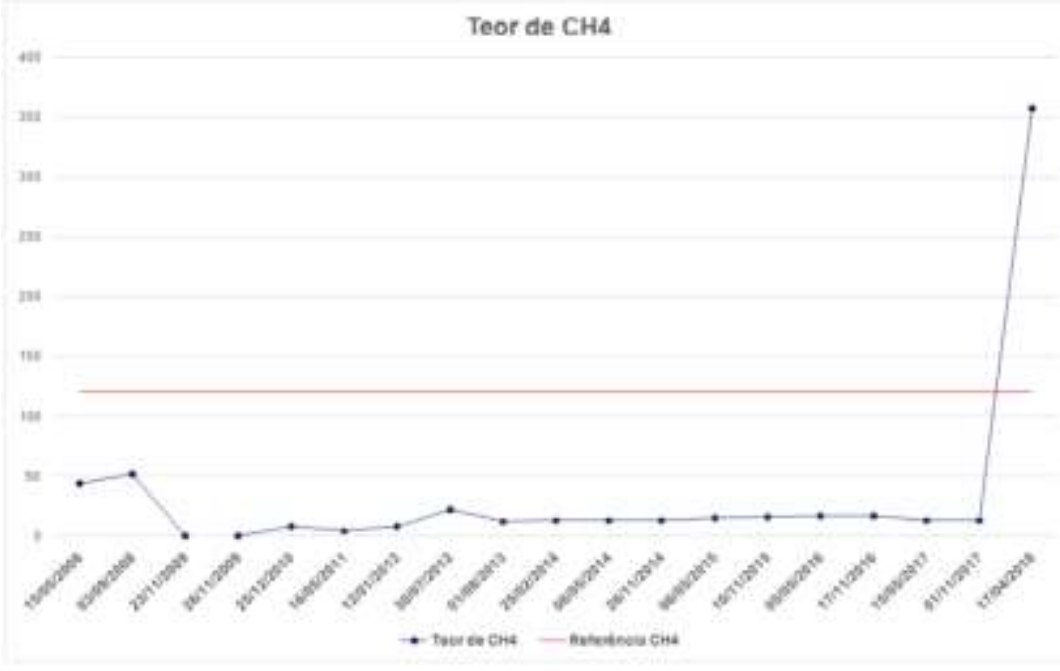

b)

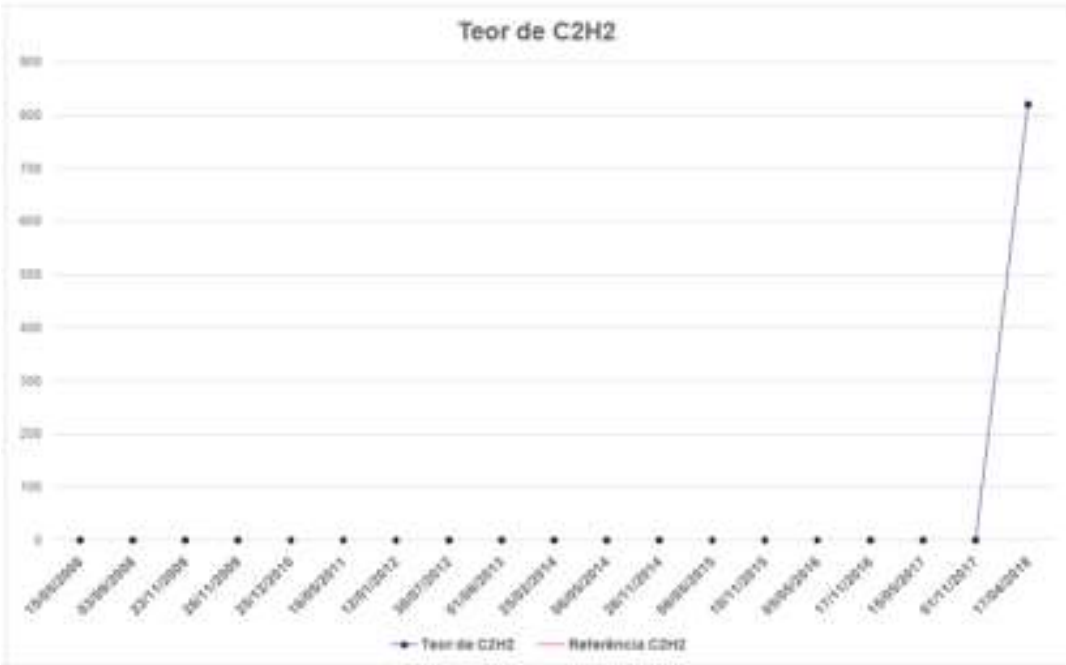

c)

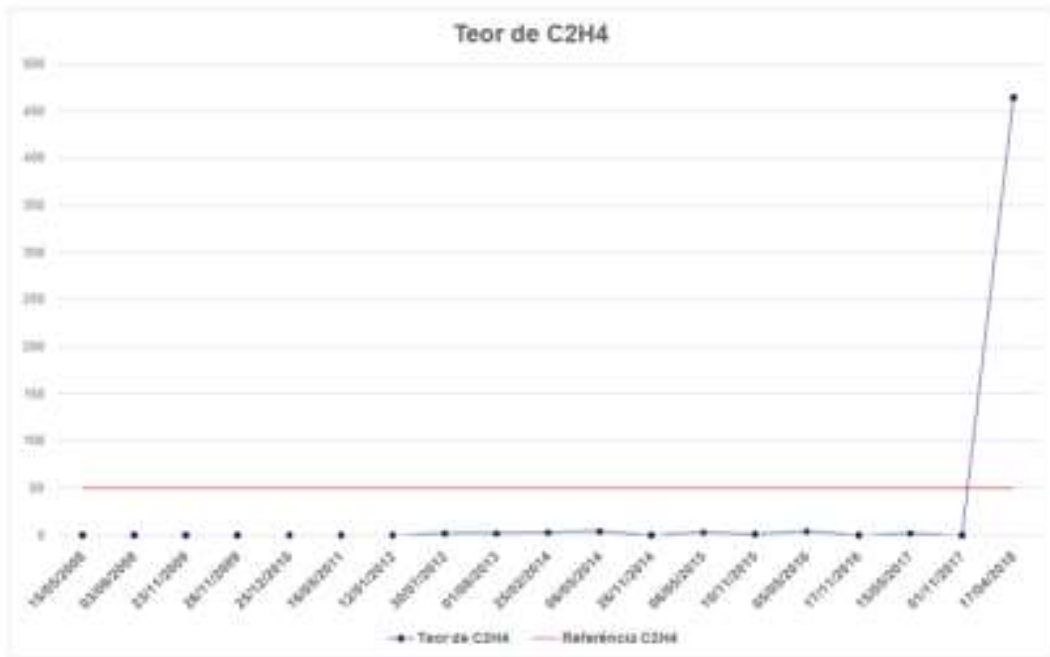

d) 
Research, Society and Development, v. 10, n. 6, e18510615460, 2021

(CC BY 4.0) | ISSN 2525-3409 | DOI: http://dx.doi.org/10.33448/rsd-v10i6.15460

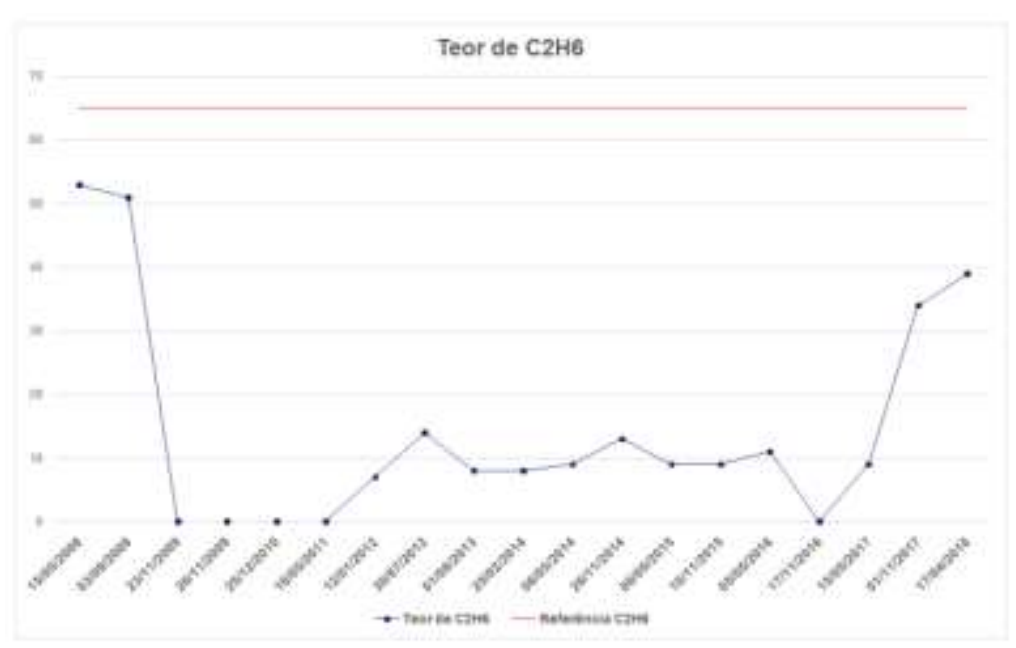

e)

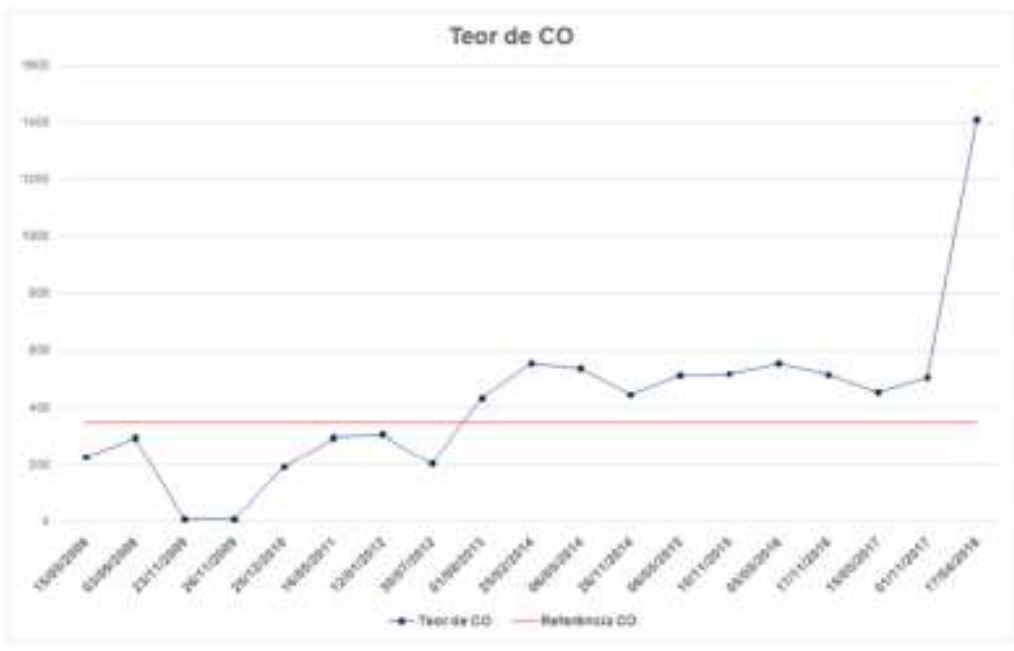

f)

Fonte: Góes (2021).

A Figura 3 (a) indica os valores das concentrações de $\mathrm{CO}_{2}$. Vê-se que a concentração do dióxido de carbono teve uma baixa em 03/09/2008 e uma alta em 16/05/2011, a partir de onde esteve acima dos valores de referência. A concentração de $\mathrm{O}_{2}$ na Figura 3 (b) não é levada em conta para diagnósticos de falta de transformadores segundo a norma IEEE C.57.104. Assim como a concentração de $\mathrm{O}_{2}$, a concentração de $\mathrm{N}_{2}$ na Figura 3 (c) não é levada em conta para diagnósticos de falta de transformadores segundo a norma IEEE C.57.104. A Figura 3 (d) indica e evolução das concentrações totais de gases, ou seja, gases combustíveis, e de gases não combustíveis como o oxigênio e o nitrogênio. Por fim, a Figura 3 (e) mostra os valores de concentração totais de gases combustíveis dissolvidos. Percebe-se que os valores de TDCG se mantiveram abaixo dos valores de referência até 01/11/2017. 
Research, Society and Development, v. 10, n. 6, e18510615460, 2021

(CC BY 4.0) | ISSN 2525-3409 | DOI: http://dx.doi.org/10.33448/rsd-v10i6.15460

Figura 3. Amostras coletadas das concentrações de Dióxido de Carbono, Oxigênio, Nitrogênio, TCG, TDCG do transformador SUTF6-03.

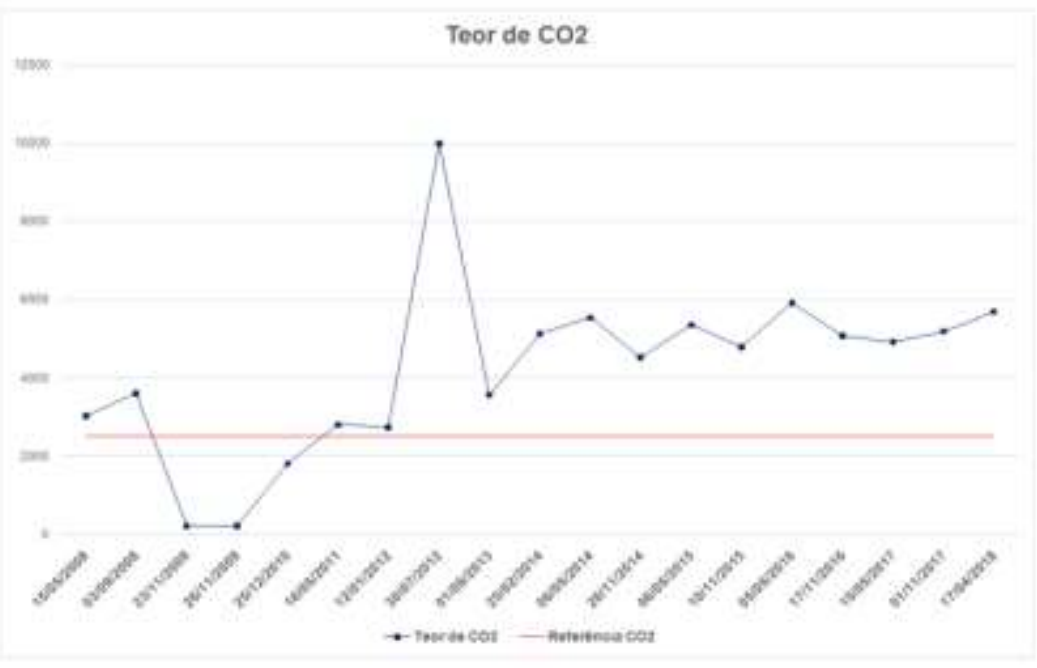

a)

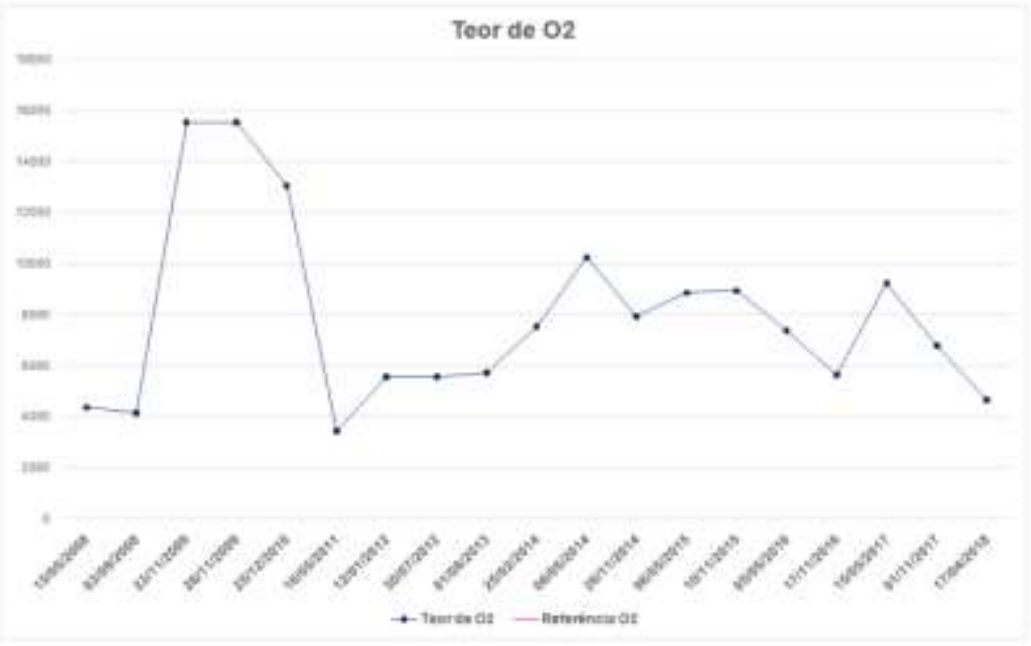

b)

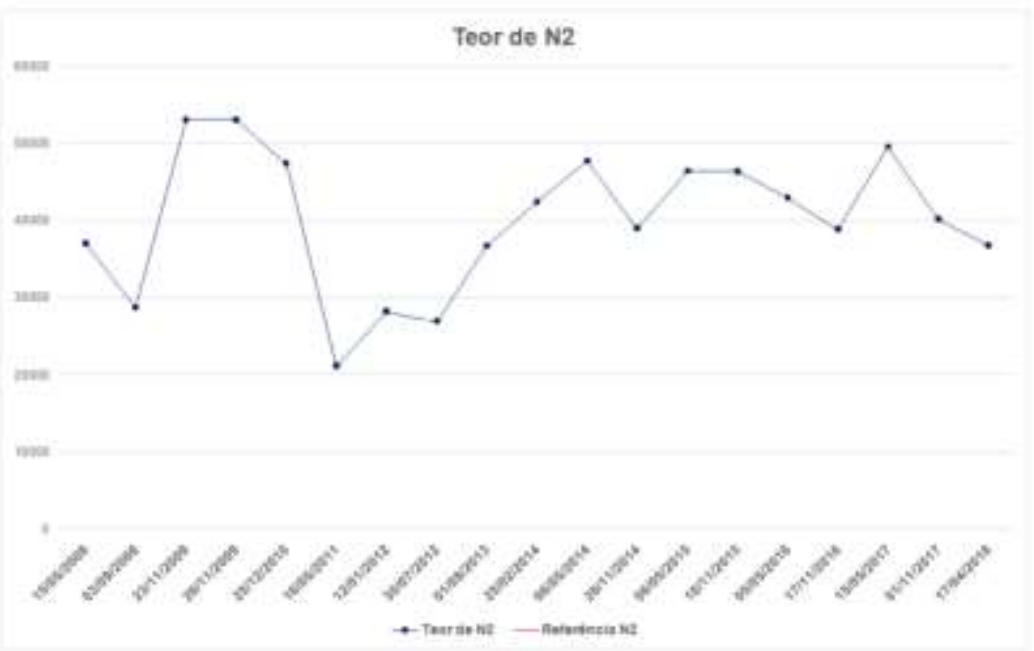

c) 
Research, Society and Development, v. 10, n. 6, e18510615460, 2021

(CC BY 4.0) | ISSN 2525-3409 | DOI: http://dx.doi.org/10.33448/rsd-v10i6.15460

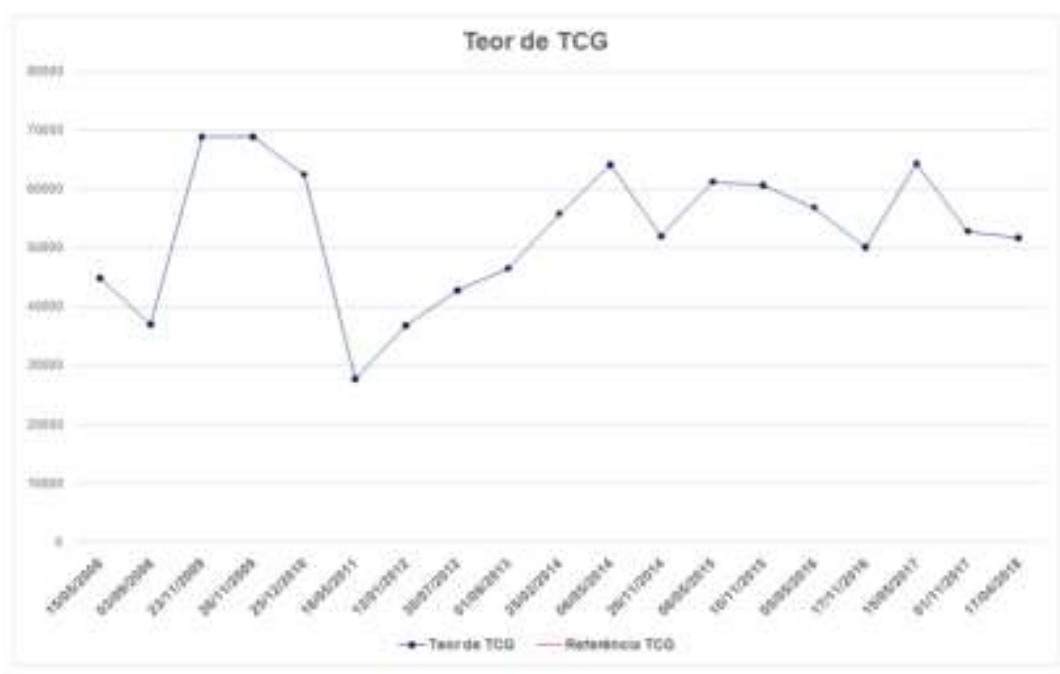

d)

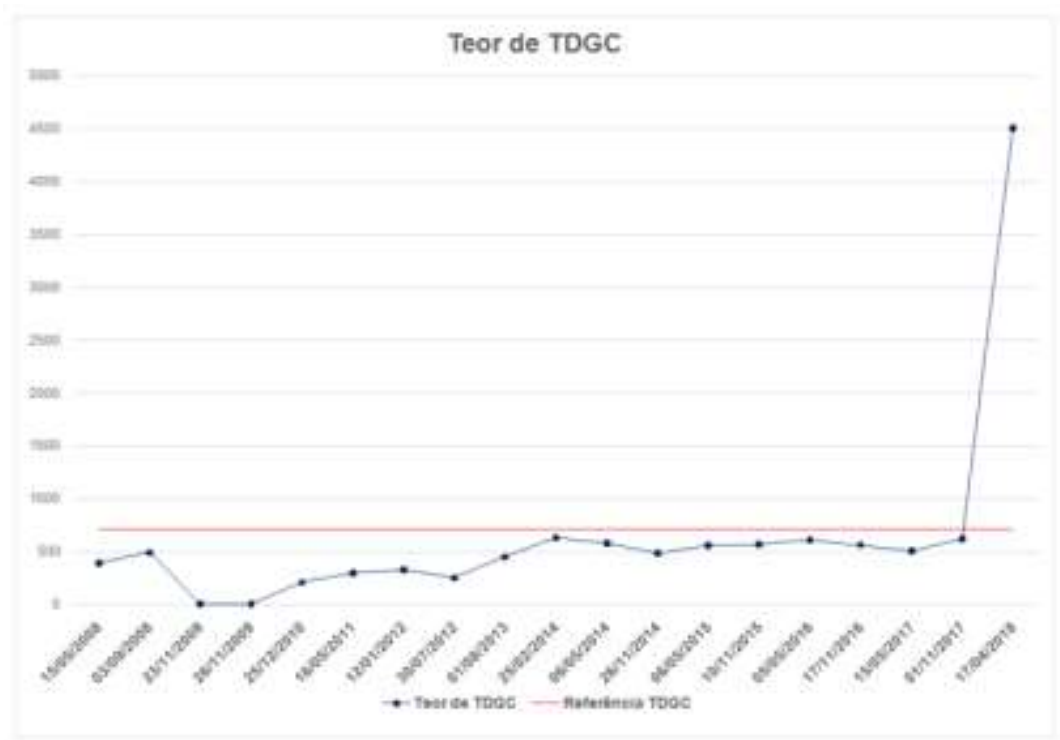

e)

Fonte: Góes (2021).

Por meio dos gráficos feitos com os dados enviados pelo laboratório da empresa, pode-se ver que há uma alta taxa de crescimento nos valores de ppm (parte por milhão) do Hidrogênio $\left(\mathrm{H}_{2}\right)$, do Metano $\left(\mathrm{CH}_{4}\right)$, do Acetileno $\left(\mathrm{C}_{2} \mathrm{H}_{2}\right)$, do Etileno $\left(\mathrm{C}_{2} \mathrm{H}_{4}\right)$ e do Total de Gases Combustíveis (TDGC).

Comparando as Tabelas 1 e 2, nota-se a diferença das concentrações de gases combustíveis nas duas amostras. Em vista disso, para o diagnóstico, estabeleceu-se razões entre as concentrações dos gases combustíveis presentes no transformador, conforme a Tabela 3. 
Research, Society and Development, v. 10, n. 6, e18510615460, 2021

(CC BY 4.0) | ISSN 2525-3409 | DOI: http://dx.doi.org/10.33448/rsd-v10i6.15460

A Tabela 1 mostra os valores das concentrações dos gases na amostra de 24/11/2017, a última coleta antes do sinistro.

Tabela 1. Concentrações dos gases da amostra de 24/11/2017.

\begin{tabular}{cc}
\hline Ensaios Realizados & Resultado [ppm] \\
\hline Hidrogênio $\left(\mathrm{H}_{2}\right)$ & 74 \\
Oxigênio - $\left(\mathrm{O}_{2}\right)$ & 6792 \\
Nitrogênio - $\left(\mathrm{N}_{2}\right)$ & 40245 \\
Metano $\left(\mathrm{CH}_{4}\right)$ & 13 \\
Monóxido de Carbono $(\mathrm{CO})$ & 505 \\
Dióxido de Carbono $\left(\mathrm{CO}_{2}\right)$ & 5202 \\
Etileno $\left(\mathrm{C}_{2} \mathrm{H}_{4}\right)$ & 0 \\
Etano $\left(\mathrm{C}_{2} \mathrm{H}_{6}\right)$ & 34 \\
Acetileno $\left(\mathrm{C}_{2} \mathrm{H}_{2}\right)$ & 0 \\
Total de Gases 52865 & 52865 \\
Total de Gases Combustíveis 626
\end{tabular}

Fonte: Góes (2021).

A Tabela 2 indica os valores das concentrações dos gases na amostra de 20/04/2018, a primeira coleta após o sinistro.

Tabela 2. Concentrações dos gases da amostra de 20/04/2018.

\begin{tabular}{|c|c|}
\hline Ensaios Realizados & Resultado [ppm] \\
\hline Hidrogênio $\left(\mathrm{H}_{2}\right)$ & 1412 \\
\hline Oxigênio - $\left(\mathrm{O}_{2}\right)$ & 4677 \\
\hline Nitrogênio - $\left(\mathrm{N}_{2}\right)$ & 36854 \\
\hline Metano $\left(\mathrm{CH}_{4}\right)$ & 358 \\
\hline Monóxido de Carbono (CO) & 1410 \\
\hline Dióxido de Carbono $\left(\mathrm{CO}_{2}\right)$ & 5701 \\
\hline Etileno $\left(\mathrm{C}_{2} \mathrm{H}_{4}\right)$ & 465 \\
\hline Etano $\left(\mathrm{C}_{2} \mathrm{H}_{6}\right)$ & 39 \\
\hline Acetileno $\left(\mathrm{C}_{2} \mathrm{H}_{2}\right)$ & 822 \\
\hline Total de Gases & 51738 \\
\hline Total de Gases Combustíveis & 4506 \\
\hline
\end{tabular}

Fonte: Góes (2021). 
Tabela 3. Diagnóstico usando o método de Rogers de acordo com IEEE c.57.104.

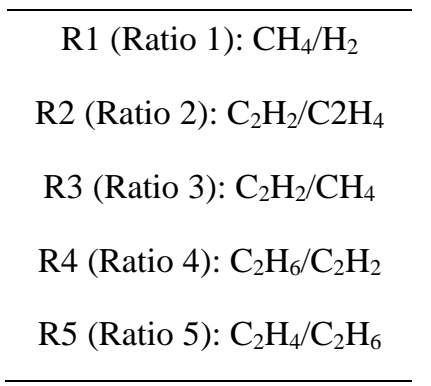

Fonte: Góes (2021).

Na Tabela 4 são apresentadas as razões do Método de Rogers usadas para diagnóstico de faltas de acordo com o IEEE C57.104.

Tabela 4. Razões de Rogers para diagnóstico de faltas.

\begin{tabular}{llllc}
\hline Caso & R1 & R2 & R5 & Diagnóstico de faltas \\
\hline 0 & $>0,1$ e $<1,0$ & $<0,1$ & $<1,0$ & Unidade Normal \\
1 & $<0,1$ & $<0,1$ & $<1,0$ & Arc o de baixa densidade de energia \\
2 & 0,1 até 1,0 & 0,1 até 3,0 & $>3,0$ & Descarga de alta densidade de energia \\
3 & $>0,1$ e $<1,0$ & $<0,1$ & 1,0 até 3,0 & Baixa temperatura térmica \\
4 & $>0,1$ & $<0,1$ & 1,0 até 3,0 & Temperatura $<700^{\circ} \mathrm{C}$ \\
5 & $>0,1$ & $<0,1$ & $>3,0$ & Temperatura $>700^{\circ} \mathrm{C}$ \\
\hline
\end{tabular}

Fonte: Adaptado de IEEE (2009).

Calculando R1, R2 e R5 e usando 5 casas decimais temos os resultados apresentado na Tabela 5.

Tabela 5. Cálculo dos R1, R2 e R3.

$\mathrm{R} 1=358 / 1412=0.25354(1)$
$\mathrm{R} 2=822 / 465=1.76774(2)$
$\mathrm{R} 5=465 / 39=11.92307(3)$

Fonte: Góes (2021).

Relacionando os resultados das Equações 1, 2 e 3 com a Tabela 4, pode-se afirmar que a falta ocorreu por conta de descargas parciais de alta intensidade de energia.

De acordo com as normas que Eletrobras Eletronorte usou como base para o diagnóstico do sinistro por meio das amostras de gás foram a American Society for Testing and Materials (ASTM D 3612 de 2002, Método C) e International Electrotechnical Commission (IEC 60599 de 2007).

Norma/instrução técnica utilizados: Análise de gases dissolvidos - Método C da ASTM D3612 de 2002 (09); interpretação da análise de gases dissolvidos - IEC 60599 de 2007; ITM EM-0002. Instrumentos utilizados: Cromatógrafo gasoso AGILENT modelo 7890A com auto-amostrador tipo HEADSPACE modelo Combipal. (ELETRONORTE, 2018).

Percebe-se que o Método de Rogers da IEEE C.57.104 proporcionou o mesmo diagnóstico, validando assim o método como eficaz, e uma das provas foi a avaria sofrida pelo relé 63 do SUTF6-03 em decorrência da descarga parcial de alta intensidade de energia, o que dificultou a restrição de possíveis causas da falta ocorrida no transformador. Na Figura 4 pode-se 
ver o estrago causado pela descarga parcial nos contatos do Relé 63 do Transformador.

Figura 4. Relé Buchholz do transformador SUTF6-03 após o sinistro.

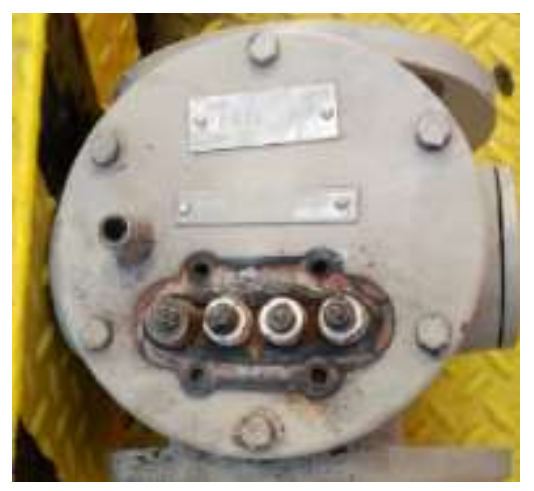

Fonte: Autores.

Foi feita uma raspagem nos contatos do relé, pois o mesmo estava com os fios nos contatos por conta da descarga parcial.

A realização de uma análise de gases isoladamente nem sempre permite um diagnóstico completo das condições do transformador, é necessário que se levem em conta as análises de falhas anteriores, no sentido de se avaliar o desenvolvimento de uma falha. A confiabilidade e o histórico do equipamento pesam na hora de se diagnosticar uma falta e também nas tomadas de decisão em relação a tirar ou não de operação o transformador. De acordo com a Figura 3, vê-se que o transformador SUTF603 não possuía um histórico de faltas por conta de gases combustíveis.

\section{Conclusão}

No início do trabalho, viu-se a necessidade de métodos capazes de diagnosticar com precisão os motivos das faltas que ocorrem em transformadores de potência. Portanto, o Método de Rogers mostrou-se capaz de resolver essa problemática, onde por meio do estudo de caso fez-se o levantamento de amostras de óleo do transformador STUF6-03 para serem analisadas e se chegar no diagnóstico correto da falta que levou o equipamento a sair de operação.

O primeiro objetivo específico era descrever a teoria de transformadores de potência. No presente trabalho vimos a teoria geral de transformadores, bem como o seu funcionamento e os métodos de proteção mais usados para transformadores de potência. O segundo objetivo específico era apontar os principais métodos de análise de óleo mais utilizados para diagnóstico e monitoramento de transformadores. Esse assunto foi coberto abordando diversos métodos de análise de óleo e os tipos de faltas que ocorrem nos transformadores de acordo com as concentrações de gases presentes no equipamento. $\mathrm{O}$ terceiro objetivo específico era comprovar a eficácia do Método de Rogers para diagnóstico de faltas de transformadores.

Usando as concentrações de gases enviadas pelo laboratório LACEN, viu-se que o Método de Rogers se provou capaz de diagnosticar com precisão a falta ocorrida no transformador SUTF6-03 da UHE Samuel. A coleta dos dados usados no trabalho se deu com o envio do LACEN das amostras de óleo do transformador, onde fez-se um compilado por meio de gráficos, com os dados dessas amostras dos últimos 10 anos. Por meio desses gráficos foi possível observar a crescente concentração de gases combustíveis no transformador em relação aos seus valores normais.

Como sugestão de trabalho futuro pode ser abordada a análise de óleo dos transformadores não só como método de diagnóstico, mas também como método de prevenção de faltas. Para isso deveria haver um estudo mais aprofundado das análises de óleo como técnicas preditivas. Em vista disso, percebe-se uma importância em trabalhos futuros nos quais englobam aprofundamento no estudo sobre proteção de transformadores, principalmente sobre os relés 63 e 87, e uma análises de óleo do 
transformador como método usado para prevenção de faltas.

\section{Agradecimentos}

Aos professores Antonio Carlos Duarte Ricciotti e Viviane Barrozo da Silva pela dedicação e pelo apoio. Ao meu orientador de estágio na Eletronorte Robinson Percy Holder e à equipe da engenharia elétrica da UHE Samuel por compartilharem do seu conhecimento.

\section{Referências}

Aguiar, E. R. de. (2007). Análise de resposta em frequência aplicada em transformadores de potência. 207. (Dissertação de Mestrado). Escola de Engenharia, Universidade Federal de Mina Gerais, Belo Horizonte.

Almeida, L. S., Muniz P. R. (2020). Análise de desempenho do transformado de potência isolado a óleo vegetal em relação ao transformador de potência is olado a óleo mineral. Revista 29. Revista Ifes Ciência, 6(2), 28-41. https://doi.org/10.36524/ric.v6i2.629

Mayor, G. A. (2020). Ferramenta de software para estimar o índice de saúde em transformadores de potência. e-Gnose, 18.

Barbosa, F. R. (2013). Diagnóstico de falhas incipientes a partir das propriedades físico-químicas do óleo isolante em transformadores de potência como método alternativo à análise de gases dissolvidos. 133. (Tese de Doutorado). Centro de Tecnologia, Universidade Federal do Ceará.

Bechara, R. (2010). Análise de falhas de transformadores de potência. 118. (Dissertação de Mestrado). Escola Politécnica da Universidade de São Paulo. https://doi.org/10.11606/D.3.2010.tde-16082010-163504

Benedet, M. E. (2007). Otimização de um analisador de gás dissolvido em óleo de múltiplos transformadores de potência. 111. (Dissertação de Mestrado). Universidade Federal de Santa Catarina.

Caldeira, P. H. C. (2019). Diagnóstico de falha em transformador imerso a óleo isolante usando uma rede neural artificial. 71. (Monografia de Graduação). Instituto de Ciências Exatas e Aplicadas, Universidade Federal de Ouro Preto.

Chapman, S. J. (2013). Fundamentos de máquinas elétricas. (5a ed.), AMGH Editora.

Costa, J. P. M. (2018). análise de falhas em transformadores de corrente. 51. (Dissertação de Doutorado) Universidade Federal do Rio de Janeiro.

Eletronorte, E. (2018). Relatório Técnico RT034902018. 2.

Góes, H. O. (2021). Falhas Em Transformadores De Potência: Estudo De Caso Do Sutf6-03 Na Uhe Samue.

IEEE. (2009). IEEE C57.104-2008 - IEEE Guide for the Interpretation of Gases Generated in Oil-Immersed Transformers. 39.

Ikeshoji, M. A. (2020). Desenvolvimento de metodologia para diagnóstico de falhas incipientes em transformadores de potência utilizando análise de gases e aprendizado de máquina.

Jordão, R. G. (2002). Transformadores. Editora Blucher.

Leopoldino, R. (2011). Estudos sobre gases dissolvidos em óleos vegetais isolantes influência de arco elétrico e superaquecimento. 89. (Dissertação de Mestrado). Escola de Engenharia de São Carlos. 10.11606/D.18.2011.tde-02062011-141424

Lima, S. L. (2008). Diagnóstico de falhas incipientes em transformadores de potência utilizando a teoria da expansão. 94.(Dissertação de Mestrado). Universidade Federal do Maranhão, São Luis.

Lupi Filho, G. (2017). Comparação entre os critérios de diagnósticos por análise cromatográfica de gases dissolvidos em óleo isolante de transformador de potência. 148. (Dissertação de Mestrado). Escola de Engenharia de São Carlos.

Pinterest. (2020). Pinterest.com. <https: //www.pinterest.es/pin/390405861417700943/>.

Santana, T. S. D. (2018). A termografia aplicada à manutenção de transformadores de potência a seco. 126. (Dissertação de Mestrado). Escola Politécnica da Universidade Federal da Baia.

Senna, S. L. de. (2010). Computação evolucionária aplicada ao diagnóstico de falhas incipientes em transformadores de potência utilizando dados de cromatografia. 101. (Dissertação de Mestrado) Universidade Federal de Minas Gerais

Silva, D. F. F. da. (2016). Monitorização e diagnóstico da condição de transformadores de alta potência para melhorar a fiabilidade e gestão do respetivo ciclo de vida. 118. (Dissertação de Mestrado) Universidade de Lisboa

Enriquez, A. R. S. (2020). Diagnóstico de falhas em transformadores de potência através de análise de gases dissolvidos usando rede neural artificial. (Dissertação de Mestrado) Universidade Federal do Maranhão 